\title{
Body configuration in cycling affects muscle recruitment and movement pattern
}

Citation for published version (APA):

Savelberg, H. H. C. M., van de Port, I. G., \& Willems, P. J. B. (2003). Body configuration in cycling affects muscle recruitment and movement pattern. Journal of Applied Biomechanics, 19(4), 310-324.

https://doi.org/10.1123/jab.19.4.310

Document status and date:

Published: 01/01/2003

DOI:

10.1123/jab.19.4.310

Document Version:

Publisher's PDF, also known as Version of record

Document license:

Taverne

Please check the document version of this publication:

- A submitted manuscript is the version of the article upon submission and before peer-review. There can be important differences between the submitted version and the official published version of record.

People interested in the research are advised to contact the author for the final version of the publication, or visit the DOI to the publisher's website.

- The final author version and the galley proof are versions of the publication after peer review.

- The final published version features the final layout of the paper including the volume, issue and page numbers.

Link to publication

\footnotetext{
General rights rights.

- You may freely distribute the URL identifying the publication in the public portal. please follow below link for the End User Agreement:

www.umlib.nl/taverne-license

Take down policy

If you believe that this document breaches copyright please contact us at:

repository@maastrichtuniversity.nl

providing details and we will investigate your claim.
}

Copyright and moral rights for the publications made accessible in the public portal are retained by the authors and/or other copyright owners and it is a condition of accessing publications that users recognise and abide by the legal requirements associated with these

- Users may download and print one copy of any publication from the public portal for the purpose of private study or research.

- You may not further distribute the material or use it for any profit-making activity or commercial gain

If the publication is distributed under the terms of Article $25 \mathrm{fa}$ of the Dutch Copyright Act, indicated by the "Taverne" license above, 


\title{
Body Configuration in Cycling Affects Muscle Recruitment and Movement Pattern
}

\author{
H ans H.C.M. Savelberg, Ingrid G.L. Van de Port, \\ and Paul J.B. Willems \\ M aastricht $U$ niversity, The $\mathrm{N}$ etherlands
}

By manipulating trunk angle in ergometer cycling, we studied the effect of body configuration on muscle recruitment and joint kinematics. Changing trunk angle affects the length of muscles that span the hip joint. It is hypothesized that this affects the recruitment of the muscles directly involved, and as a consequence of affected joint torque distributions, also influences the recruitment of more distal muscles and the kinematics of distal joints. It was found that changing the trunk from an upright position to approximately $20 \mathrm{deg}$ forward or backward affected muscle activation patterns and kinematics in the entire lower limb. The knee joint was the only joint not affected by manipulation of the lengths of hip joint muscles. Changes in trunk angle affected ankle and hip joint kinematics and the orientation of the thigh. A similar pattern has been demonstrated for muscle activity: Both the muscles that span the hip joint and those acting on the ankle joint were affected with respect to timing and amplitude of EMG. Moreover, it was found that the association between muscle activity and muscle length was adapted to manipulation of trunk angle. In all three conditions, most of the muscles that were considered displayed some eccentric activity. The ratio of eccentric to concentric activity changed with trunk angle. The present study showed that trunk angle influences muscle recruitment and (inter)muscular dynamics in the entire limb. As this will have consequences for the efficiency of cycling, body configuration should be a factor in bicycle design.

Key Words: electromyography, biomechanics, eccentric contraction, joint kinematics

The authors are with the Dept. of Human Movement Science, Nutrition and Toxicology Research Institute (NUTRIM), Faculty of Health Sciences, Maastricht University, NL6200MD Maastricht, The Netherlands. 


\section{Introduction}

Recumbent cycling is gaining popularity (Reiser, Peterson, \& Broker, 2001), not only in high-performance human powered vehicles but also in ergometrics and rehabilitation settings (Gregor, Perell, Rushatakankovit, et al., 2002). In rehabilitation, recumbents are often preferred over standard bikes because they offer a more stable position for the patient and easier access (Gregor et al., 2002). However, there is little information as to the effects of recumbent cycling on muscle recruitment and joint loading. In human-powered vehicles, the increased efficiency of recumbents is important. The higher efficiency in recumbent cycling is mostly attributed to reduced aerodynamic losses (Gross, Kyle, \& Malewicki, 1983). However, when comparing a standard crouched racing position to an uncovered recumbent position, it is questionable whether this is the only factor. The crouched position gives the cyclist an egg-shaped body form, which is known to reduce the drag coefficient. In the uncovered recumbent position, the cyclist becomes rather a spoiler, which may not necessarily have a positive effect on drag coefficient. Therefore, to explain the positive effects on performance of recumbent cycling compared to the standard crouched racing position, other factors that enhance the human contribution to the bicycle should be considered as well.

The standard cycling position has been studied extensively; effects of numerous factors on muscle recruitment, dynamic and kinematic patterns, and energy expenditure have been described, i.e., cadence (Ericson, Nisell, Arborelius, \& Ekholm, 1985; MacIntosh, Neptune, \& Horton, 2000; Marsh \& Martin, 1995; Neptune, Kautz, \& Hull, 1997), body orientation (Brown, Kautz, \& Dairaghi, 1996; Li \& Caldwell, 1998), seat tube height (Jorge \& Hull, 1986), and chain disc design (Neptune \& Herzog, 2000). For recumbents this information is generally missing. For the optimization of human powered vehicles, and for designing effective rehabilitation protocols and setups, this is a handicap. Differences between the recumbent and upright positions are determined by two factors: leg orientation and body configuration. Body configuration refers to the angle between the trunk and the line connecting the center of the hip joint and the crank axle. Leg orientation refers to the angle between the horizontal and the line connecting the center of the hip joint and the crank axle. Both in recumbent and upright cycling, these factors can be manipulated independently.

Body configuration affects the length of the muscles that span the hip joint, and therefore the force these muscles can contribute to cycling. Van Ingen Schenau, Boots, de Groot, Snackers, and van Woensel (1992) have analyzed the association between recruitment of muscles in multibody systems and directing an external force. They concluded that tuning of mono- and biarticular muscle activation is required in order to optimally direct external force. It can be hypothesized that changing the length of muscles spanning the hip joint will affect intermuscular coordination and the optimal direction of force on the pedal, thus contributing to changes in efficiency. Leg orientation will affect the contribution of gravity to the movement. The cycling movement is accomplished by muscular work and by gravitational work. Optimizing the coordination between these contributors can reduce the amount of muscular work and may improve performance. Manipulation of leg orientation affects the crank angle, and thus the joint angles and muscle lengths 
with respect to the pattern of positive and negative gravitational work. Therefore this factor can also be expected to affect intermuscular coordination.

The redundant number of muscles in the limb and the lack of recruitment rules applied by the neurological system makes it impossible to predict just how muscle will be recruited when either body configuration or leg orientation are manipulated. Moreover, in the vast amount of cycling studies these factors have not been considered independently. To unravel the effects of both factors on muscle recruitment, it is necessary to consider them independently. Insight into the effects of body configuration and leg orientation on cycling performance will help to optimize recumbent bicycles both for high performance and for use in rehabilitation.

The aim of this study was to investigate the effect of body configuration on muscle recruitment. Information on body configuration is relevant for both the upright and the recumbent position. To prevent confusion with effects of leg orientation, this study considers the effects of manipulated body configuration in the standard upright leg orientation. It is hypothesized that the mere manipulation of the hip joint angle will affect the timing and the amount of activation in more distal leg muscles. As a consequence of adaptations in muscle recruitment, kinematics of knee and angle joint angles and the length of muscle during activity will become affected too.

\section{Methods}

Eight cyclists, age $22.3 \pm 1.2$ years, participated in this study. They were familiar with cycling through daily and recreational activities. All participants gave their informed consent.

Following a 10-min warm-up, a short maximal ergometer test was conducted in order to determine maximal power. This maximal test was an incremental cycle test, starting with a 2-min stage at $100 \mathrm{~W}$ for men and $50 \mathrm{~W}$ for women. The workload was increased by $25 \mathrm{~W}$ every minute until exhaustion or until the participant could no longer maintain a cadence of $70 \mathrm{rpm}$. This short test was chosen because we only needed to determine the maximal power that participants could generate for several seconds. The test was used to normalize the ergometer load that the cyclists were to deal with during the test. Another reason for choosing a short test is to avoid fatigue. Based on the maximal power, we calculated the load during the test as being $80 \%$ of maximal power.

Three different body configurations were randomly imposed: upright, flexed forward, and extended backward. The orientation of the leg, being the angle of the seating tube, was constant (Figure 1). A custom-designed rest supported the trunk in both the forward and backward configurations. The arms were not used to support the trunk. There were no mechanical restrictions to the position of the cyclists on the saddle, nor of the feet on the pedals. The cyclists were asked to maintain the same position over different conditions. This was checked a posteriori by the position of a marker attached to the greater trochanter relative to pedal axis. This check revealed no occurrence of position changes over conditions. After a short warming up and habituation to trunk orientation, the participants cycled for a few minutes at a constant cadence of $70 \mathrm{rpm}$ on the $80 \%$ power level in each trunk configuration. During the period of constant cadence, EMG and video registrations were measured. The participants were not aware of the exact measuring periods. 
Figure 1 - A stick-figure showing the leg, upper leg, pelvis, and upper body (triangle $=$ foot). Pelvis and upper body shown in upright (UC), extended backward (BC), and flexed forward (FC) configurations. Small pellets mark iliac crest, greater trochanter, lateral epicondyle, lateral malleolus, calcaneus, and pedal axis. Trunk angle $(\alpha)=$ angle on anterior side between markers on iliac crest and on greater trochanter and a horizontal line crossing the hip joint. Hip joint angle $(\beta)=$ angle on anterior side between line connecting markers on iliac crest and on greater trochanter and that connecting the markers representing the thigh (greater trochanter and lateral epicondyle).

Angle on posterior side between thigh line and a vertical line through hip joint determined thigh orientation $(Y)$. Knee joint angle $(\delta)=$ angle on posterior side between lines representing thigh and leg (lateral epicondyle and lateral malleolus). Ankle joint angle $(\varepsilon)=$ angle on anterior side between line of leg and line connecting heel marker and marker on rotation axis of pedal. Orientation of foot $(\varsigma)=$ angle between a horizontal line and line connecting heel marker and marker on rotation axis of pedal.

To determine changes in the intermuscular coordination, EMG analyses were made of the gluteus maximus (GM), semitendinosus (ST), biceps femoris (BF), rectus femoris (RF), vastus medialis (VM), gastrocnemius lateralis (GL), soleus (SO), and tibialis anterior (TA). Using bipolar surface electrodes ( $\mathrm{Ag} / \mathrm{AgCl}$, square detection area of $1 \times 1 \mathrm{~cm}$, interelectrode distance $3.5 \mathrm{~cm}$ ), we recorded EMG signals for 15 seconds. The skin was shaved and cleansed with alcohol. The signal was differentiated and preamplified (input impedance $>500 \mathrm{M} \Omega, \mathrm{CMRR}>110 \mathrm{~dB}$, noise $<2: \mu \mathrm{V}$ rms $0.1-10 \mathrm{~Hz}$, gain factor $100 \mathrm{x}$ ) close to the electrodes. Subsequently it was amplified (K_lab, Amsterdam; gain factors 250-10,000x) and sampled at $1000 \mathrm{~Hz}$. A goniometer (Penny \& Gilles) applied to the knee was used to discriminate subsequent knee joint cycles.

Off-line the EMG data were rectified and filtered. A second-order Butterworth filter was used (cutoff frequency $15 \mathrm{~Hz}$ ) to obtain linear envelopes. Based on the goniometer signal, the linear envelopes of EMG data could be separated in single cycles representing the activity during one pedaling cycle. The goniometer displayed a sinusoidal signal, with maximal values occurring at top dead center of the cycle. Subsequent maximal values of the goniometer signal were used to separate subsequent cycles. Using an interpolation routine, we set the number of data points in each cycle to 100. Subsequently, linear envelopes of EMG data for different cycles were averaged. 
To enable comparison between cyclists, we normalized the mean linear envelopes of each muscle in the amplitude domain to the mean value of the muscle while the participant was cycling in the upright configuration. Based on the normalized and averaged linear envelopes, the EMG signals were parameterized for each participant and each condition. Duration and onset of the active phase of a muscle and the instant at which maximal activity occurred were considered as parameters characterizing the timing of muscle activity. A muscle was considered active if the processed EMG signal was higher than $20 \%$ of the maximal value in any of the three conditions. To assess changes in intensity of activity, we determined the mean EMG amplitude over a cycle and the integrated EMG over the period during which an EMG-signal exceeded the threshold. The integrated EMG was calculated as the surface under the linear envelope during the period of increased activity.

To assess kinematic adaptations to different trunk angles, we made a 4-s video recording $(50 \mathrm{~Hz})$ which was part of the 15 seconds of EMG registration. Reflecting markers $(\varnothing 2 \mathrm{~cm})$ were placed on the most lateral point of the iliac crest, on the greater trochanter, on the lateral epicondyle, on the lateral malleolus, on the calcaneus of the left leg, and on the rotation axis of the left pedal. Based on digitized marker positions, we assessed the angles of hip, knee and ankle joint, as well as the trunk and the foot with respect to a horizontal line and the thigh and the foot with respect to a vertical line (Figure 1).

The marker on the axis of the pedal was used to identify separate cycles in the kinematic data. Again an interpolation routine was used to normalize each cycle to 100 data points and allow averaging over subsequent cycles. Based on the averaged and normalized cycles, ranges of joint angles and of minimal, maximal, and averages joint angles were calculated.

To assess changes in the length ranges of a particular muscle, we used a model of Hawkins and Hull (1991). Based on averaged hip, knee and ankle joint angles, we calcuated the length ranges of the muscles during a cycle for each condition. These calculations showed whether a muscle was used in a more or a less extended length range when trunk angle was manipulated. The muscle lengths were expressed as ratio of the average length in the upright configuration. Combining these length ranges with data on EMG activity showed whether a muscle was activated during the lengthening or shortening of the muscle-tendon complex.

Differences in electromyographic and kinematic parameters were tested using a one-way repeated-measures analysis of variance. In this test, body configuration was considered as an independent within-subject factor. For pairwise comparison of two body configuration, the LSD approach was used as a post hoc test. Statistical significance was set at $p<0.05$.

\section{Results}

The trunk orientation was on average 18.6 deg extended backward and $22.3 \mathrm{deg}$ flexed forward relative to the upright position. Maximal power varied between $250 \mathrm{~W}$ and $425 \mathrm{~W}(321.9 \pm 39.0)$. The imposed $80 \%$ power ranged between $200 \mathrm{~W}$ and $340 \mathrm{~W}$.

Averaged linear envelopes (Figure $2 \mathrm{a}-2 \mathrm{~h}$ ) represent activity patterns for each muscle for the three trunk conditions. Trunk angle significantly affected the average muscle activity throughout a movement cycle of seven (BF, ST, GM, RF, TA, 

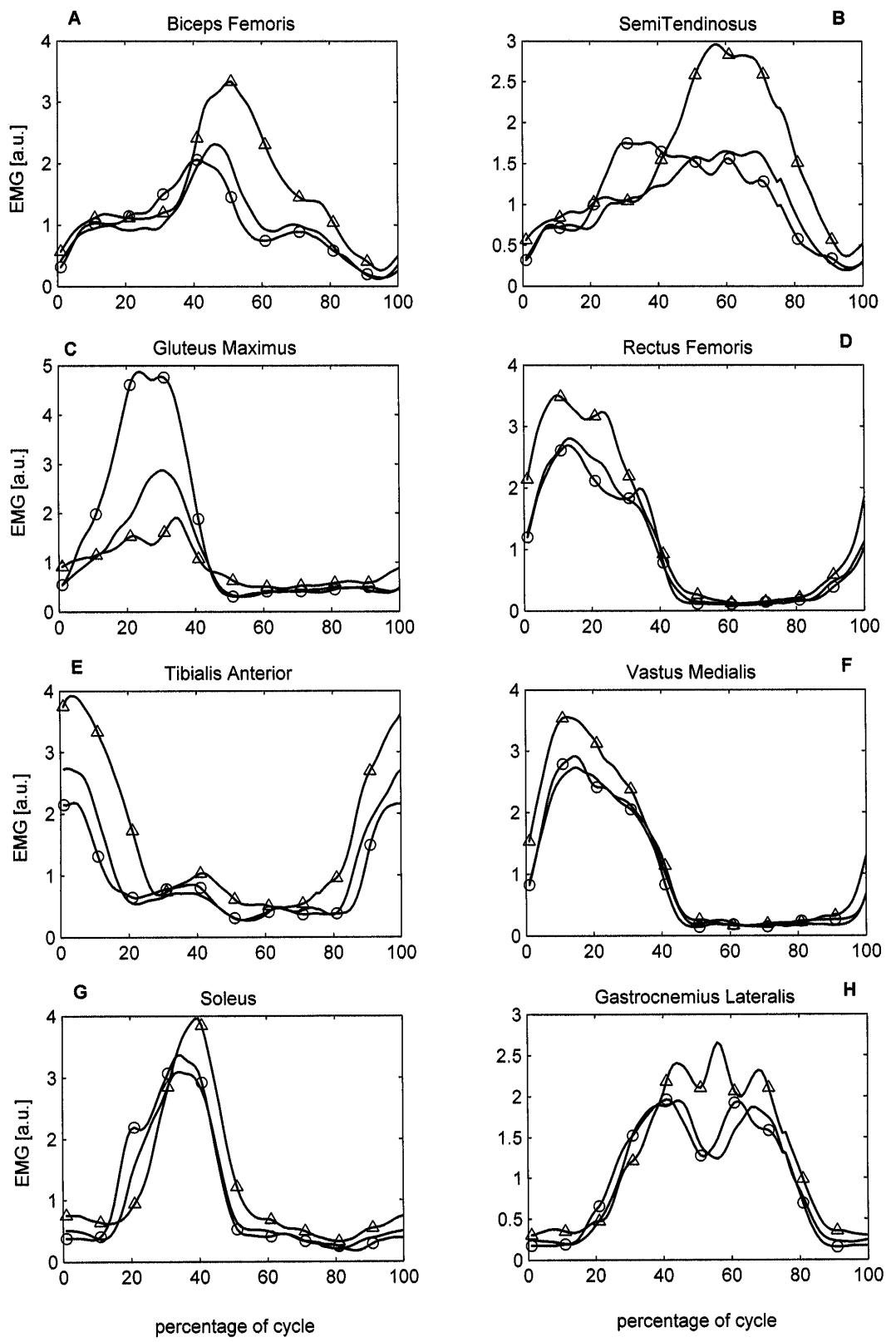

Figure 2 - Electromyographic activity of 8 leg muscles (A, biceps femoris; B, semitendinosus; C, gluteus maximus; D, rectus femoris; E, tibialis anterior; F, vastus medialis, G, soleus; H, gastrocnemius lateralis) as a function of $\%$ of full cycle for 3 trunk configurations: upright (line w/o marks); flexed $20^{\circ}$ forward $(\bigcirc)$; extended $20^{\circ}$ backward $(\Delta)$. EMG signals were rectified and filtered, averaged over several pedal cycles for each cyclist for each condition. Averaged EMG envelopes for different conditions of one cyclist for each muscle were normalized to the averaged value in the upright configuration. Finally, normalized patterns were averaged over all cyclists. 

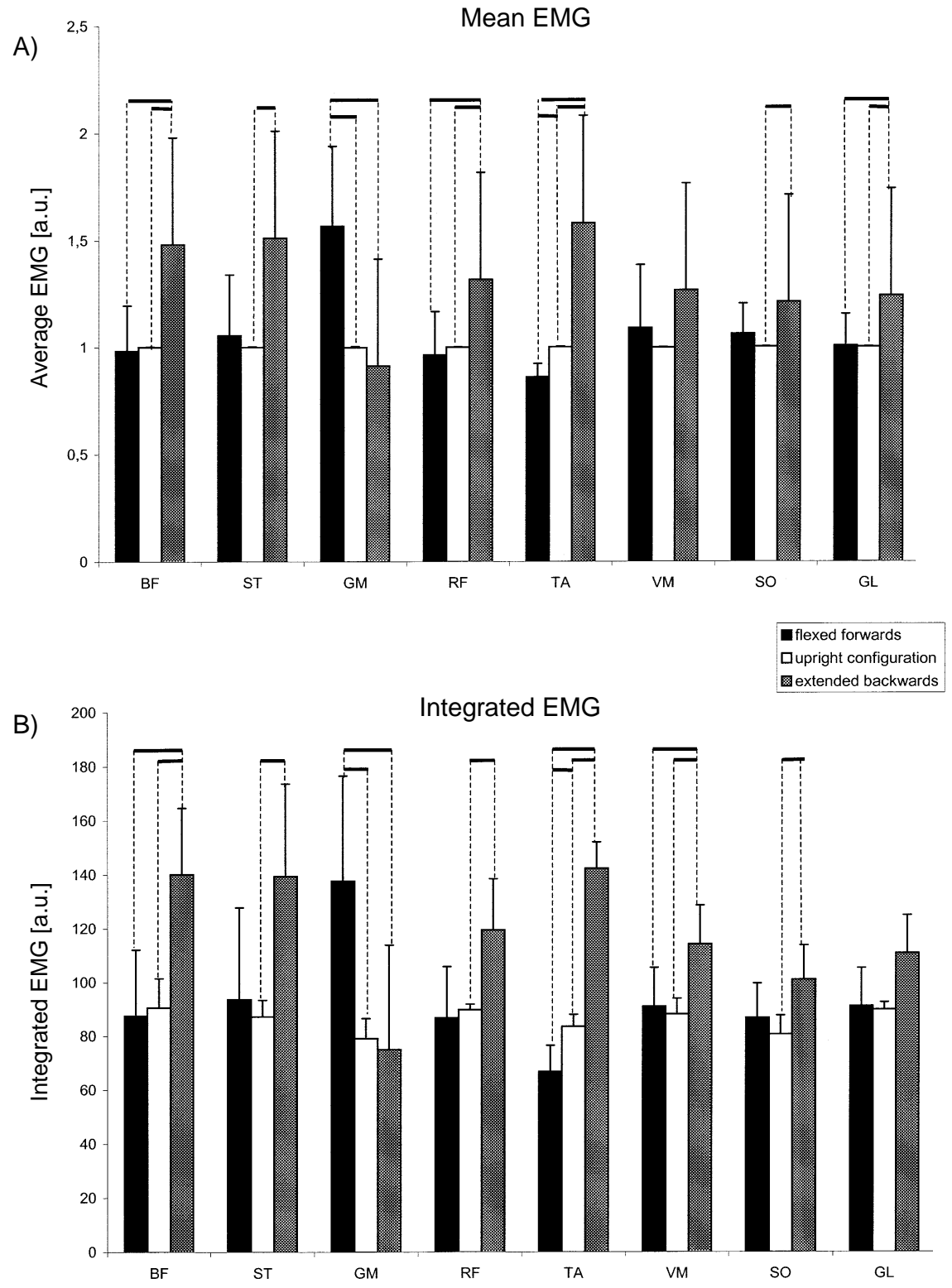

Figure 3 - Electromyographic parameters for the 8 muscles considered in 3 body configurations. Each panel shows 8 groups of 3 bars. Each group of 3 bars represents a muscle; the 3 bars represent the 3 configurations. A significant $(p<0.05)$ difference in pairwise comparison of 2 of the 3 configurations is shown by a horizontal bar above the corresponding pairs. A: mean EMG amplitude over a pedal cycle; B: integrated EMG when the amplitude exceeded $20 \%$ threshold; C: onset of muscle activity, the instant at which EMG amplitude scaled the $20 \%$ threshold (cycle starts at top dead center; negative values refer to instants before top dead center); D: period when the activity exceeded $20 \%$ threshold; E: instant when the amplitude reached it maximal value. ( $C, D, E$ continued on next page) 


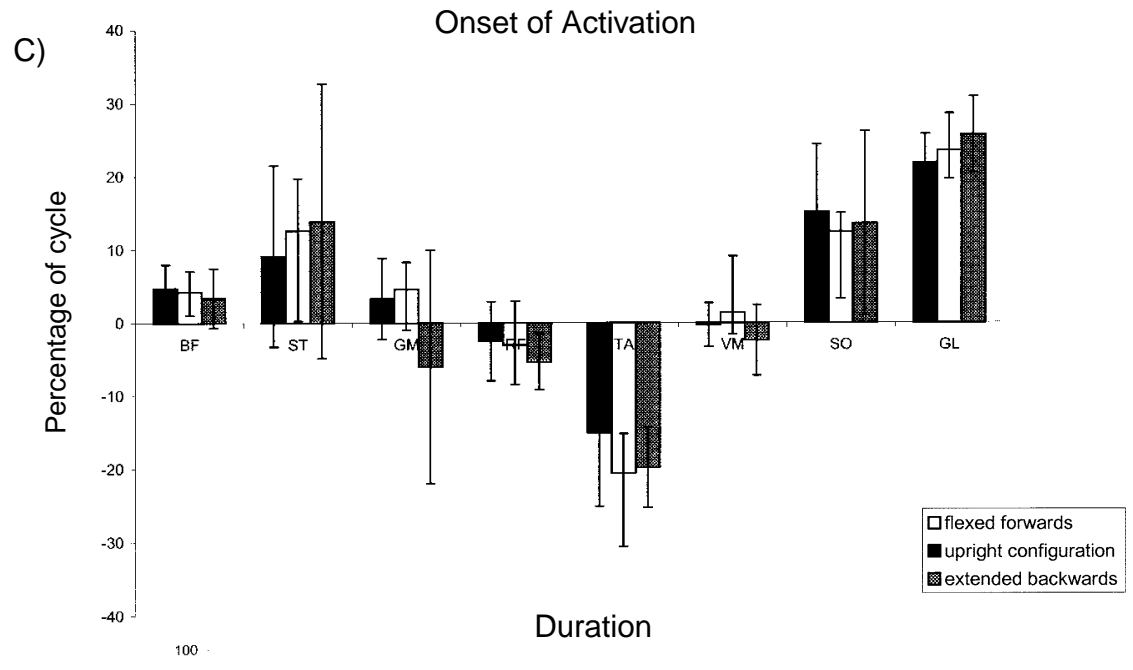

D)

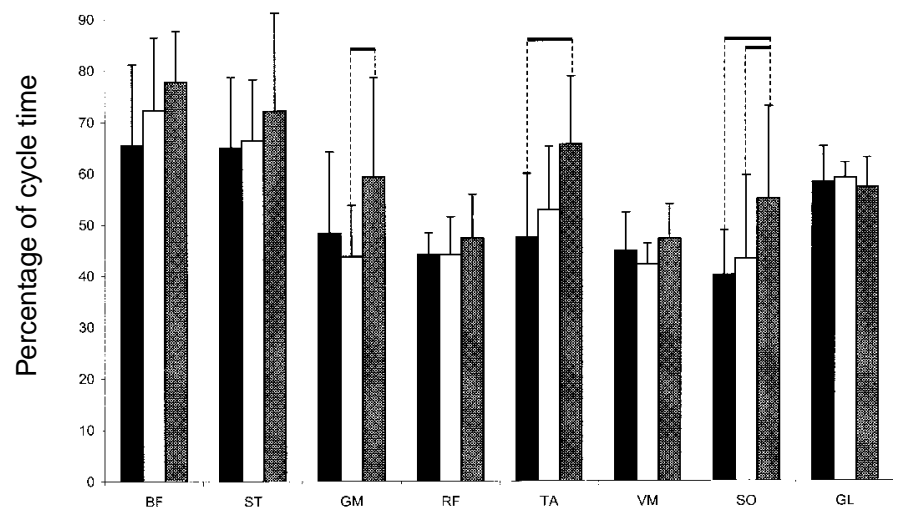

E)

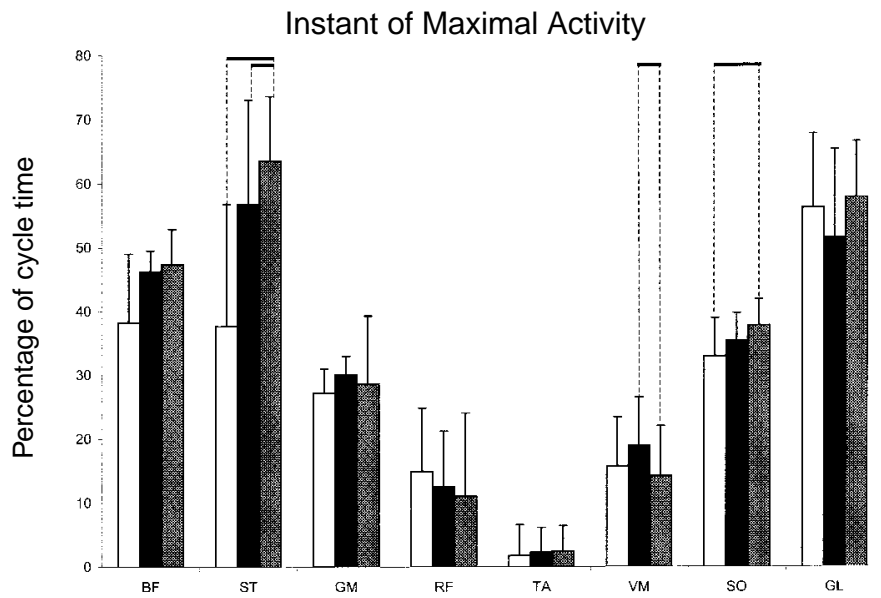

Figure 3 - Continued. 
SO, and GL) of the eight muscles considered (Figure 3a). In the GM, the average muscle activity was highest in the forward configuration. In the other muscles the activity was highest in the backward configuration. The integrated EMG was significantly affected for BF, ST, GM, RF, TA, VM, and SO. For GM the integrated EMG was highest in the forward configuration, while BF, ST, RF, and TA showed increased values in the backward configuration. In TA the integrated EMG appeared to decrease in the forward configuration compared to the upright configuration (Figure 3b).

The phase at which muscles reached the threshold differed among the muscles (Figure 3c). Onset of EMG was not influenced by trunk angle (Figure 3c). Duration of activity of GM, TA, and SO were significantly affected by trunk angle (Figure 3d); in these muscles the activity was elongated in the backward configuration. For ST, VM, and SO there was a significant effect of trunk angle on instant of maximal EMG (Figure 3e). In the forward configuration the ST reached its maximal EMG amplitude about $20 \%$ earlier in the cycle compared to both the upright and backward configurations. The VM reached its peak latest in the upright configuration. The SO had its peak shifted to a later instant with changing trunk angle from the forward via the upright to the backward configuration.

Average angles of the hip joint and the thigh changed significantly, as did the range of the hip and ankle joint angle. The hip joint angle shifted $38 \mathrm{deg}$ to more extension when changing from the forward to the backward configuration. Additionally, in the backward configuration the range of the hip joint angle was smaller than in the forward configuration (Figure 4b). The thigh angle ranged between 33 and $73 \mathrm{deg}$ in the backward configuration, and between 25 and $67 \mathrm{deg}$ in the forward configuration (Figure 4c). The average ankle joint range was significantly increased in the backward configuration (Figure 4e). No statistically significant effects on knee joint angle (Figure 4d) or foot orientation (Figure 4f) were found.

Trunk angle affected the length range of most muscles studied (Figure 5a5h). The only length ranges not affected were the monoarticular VM and the short head of the BF. For both biarticular hip extensors and knee flexors, the long head of the BF and the ST, the model predicted longer lengths in the forward configuration and smaller lengths in the backward configuration. Relative to the upright configuration, in the forward configuration the length of these muscle increased 3-4\%, while in the backward configuration a decrease of similar magnitude was found. The length change of the RF during cycling was $8 \%$ of the average length in the upright configuration. In the forward configuration the range was $2 \%$ smaller while in the backward configuration it was $2 \%$ larger. The TA displayed an increased length range of $7 \%$ in the backward configuration, compared to the $4.6 \%$ in the upright and $4.2 \%$ in the forward configuration.

Especially in the second part of the cycle, the muscle became longer in the backward configuration, up to $104 \%$ of the average length in the upright configuration. The SO and GL also had a larger range in the backward configuration. In these muscles the increased range was due to more shortening in the second phase of the cycle. In both muscles the range amounted to $8 \%$ of the average length in the backward configuration, and 5-6\% in the forward and the upright configurations.

Combining activity data and muscle length changes showed the effect of trunk angle on eccentric and concentric activity (Figure 5a-5h). GM, RF, VM, GL, and SO activity occurred in all three conditions while the muscles shortened. In SO the active period was extended in the backward configuration. The ST and BF 

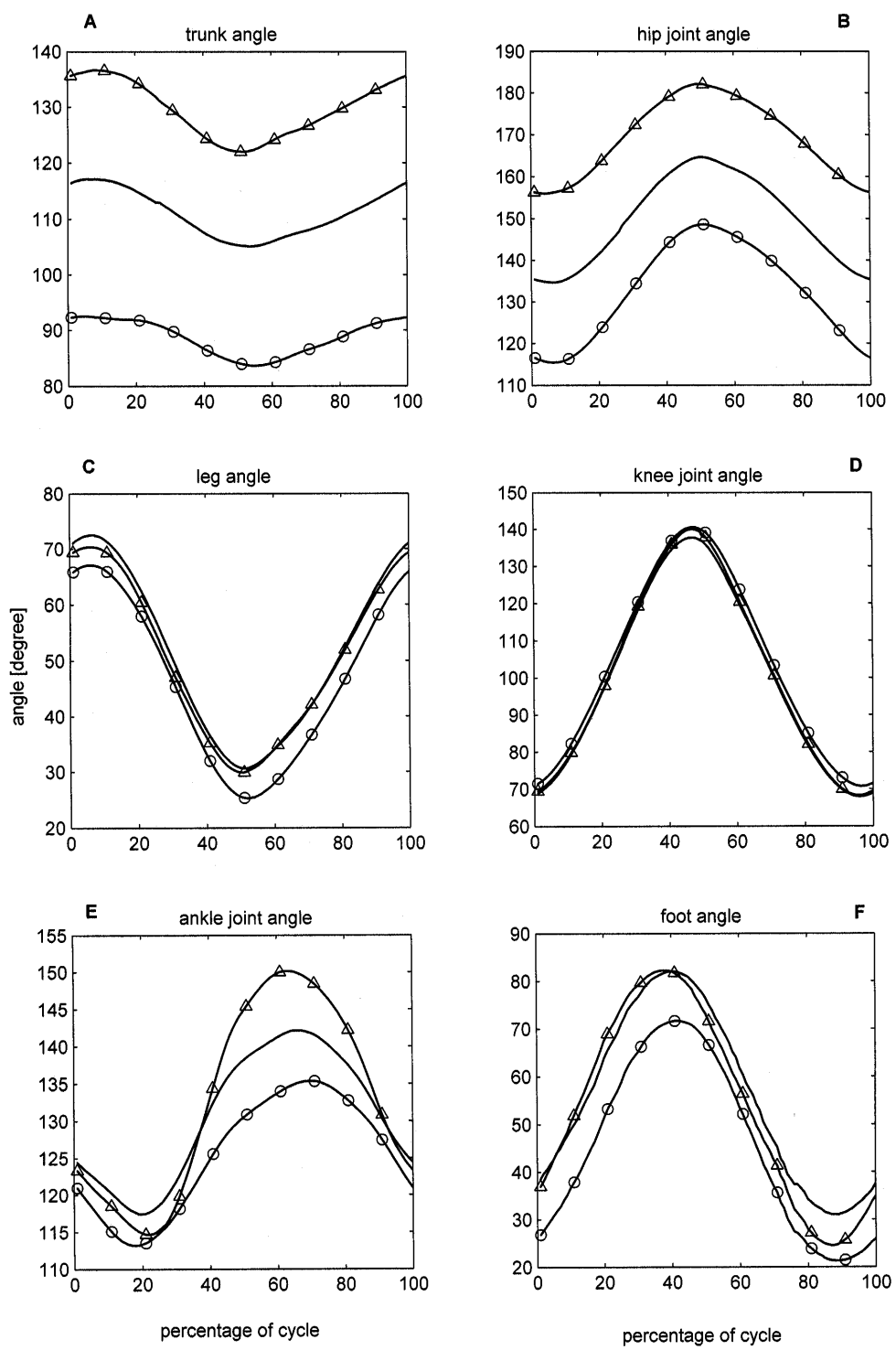

Figure $4-$ Kinematic patterns for the 3 body configurations: upright (line w/o marks); flexed $20^{\circ}$ forward $(\bigcirc)$; extended $20^{\circ}$ backward $(\Delta)$. A: trunk angle $=$ angle on anterior side between markers on iliac crest and on greater trochanter and a horizontal line crossing the hip joint; $B$ : hip joint angle $=$ angle on anterior side between line connecting the markers on iliac crest and on greater trochanter and that connecting the markers representing the thigh (greater trochanter and lateral epidcondyle); $\mathrm{C}$ : thigh orientation $=$ angle on posterior side between thigh line and a vertical line through hip joint; $D$ : knee joint angle = angle on posterior side between the lines representing thigh and leg (lateral epicondyle and lateral malleolus); E: ankle joint angle = angle on anterior side between the line of leg and line connecting heel marker and marker on rotation axis of pedal; $F$ : foot orientation $=$ angle between a horizontal line passing through pedal axis and line connecting heel marker and marker on rotation axis of pedal. 

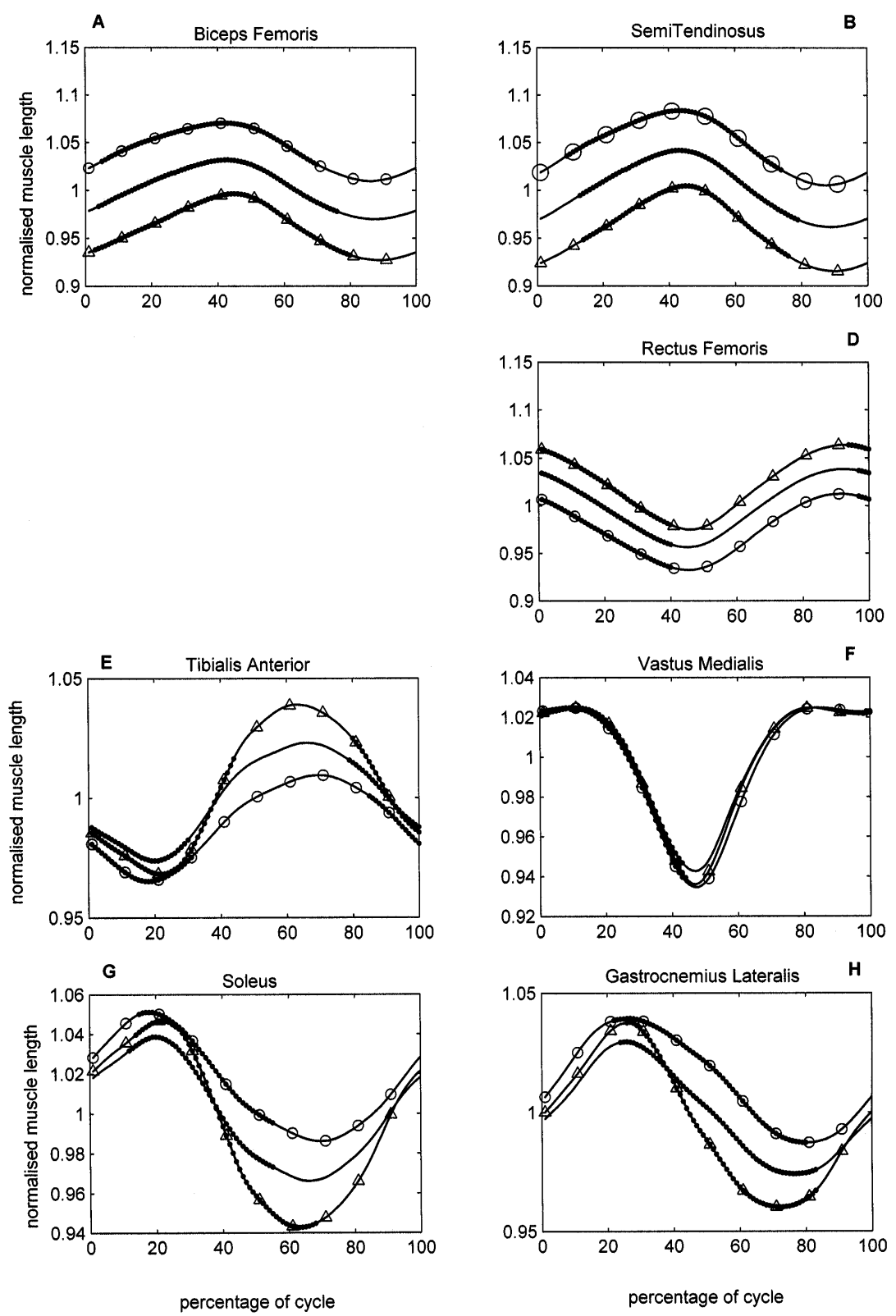

Figure 5 - Normalized muscle lengths for 7 muscles (A, biceps femoris; B, semitendinosus; D, rectus femoris; $\mathrm{E}$, tibialis anterior; $\mathrm{F}$, vastus medialis: $\mathrm{G}$, soleus; $\mathbf{H}$, gastrocnemius lateralis) in each of the 3 body configurations: upright (line w/o marks); flexed $20^{\circ}$ forward $(\bigcirc)$; extended $20^{\circ}$ backward $(\Delta)$. Normalized lengths were based on a model by Hawkins and Hull (1991). Dots on the graphs show when the EMG activity of a muscle exceeded $20 \%$ threshold. 
exhibited EMG activity in all three conditions both during elongation and during shortening. The TA displayed activity in the upright and the forward configuration only during shortening, whereas in the backward configuration the active period became increased and stretched out into the eccentric phase.

\section{Discussion}

This study evaluated the effect of body configuration on muscle recruitment and joint kinematics in cycling. So far, only from exercise physiology studies has there been evidence that trunk angle affects performance. In triathlon practice there is a trend of increasing trunk angle. Recently Garside and Doran (2000) revealed experimental evidence for the beneficial effect of this. They registered increased performance on a $10-\mathrm{km}$ run following a $40-\mathrm{km}$ bicycle ride on a steep frame configuration compared to a less steep one. Steeper frame configurations resemble more extended hip joints. In correspondence with this, Reiser et al. (2001) demonstrated a positive effect of increased body configuration angles on power output and changes in joint angle patterns. Welbergen and Clijsen (1990) found the condition comparable to the upright configuration in the present study to result in the highest $\mathrm{VO}_{2}$ max.

A better understanding of how joint angles in general and body configuration in particular can be manipulated to optimize physical performance and conditions requires insight into the mechanical base that underlies the physiological differences. The present study revealed two major findings. First, it demonstrated that in cycling, trunk angle affects the kinematics of leg movement, the activity of muscle, and the association between muscle activity and muscle length. Additionally, it demonstrated that these effects are not limited to the hip joint region, where the manipulation was applied, but effects have also been found at the knee and ankle joints and in the muscles spanning these joints.

In seven of the eight muscles considered, the EMG activity varied with trunk angle. Although the effect of increased EMG activity on muscle force cannot be quantified, the changes in EMG activity are large enough to justify the conclusion that the force generated by these muscles will be affected by manipulating the trunk angle. In addition, in five of the eight muscles-BF, ST, RF, SO, and GL(Figure 5a-5h), the length at which muscle activity occurred was influenced by trunk angle.

In spite of the fact that both the duration of electromechanical delay and the optimal length of muscle-tendon complexes are unknown, this indicates that the force generated by a muscle has been affected. Moreover, the changed association between muscle activation and muscle length affected the duration of eccentric activity in the TA and SO. In the backward configuration these muscles displayed a longer period of eccentric activity than in the other two conditions. This affects the amount of negative work to be produced by the muscles. Whereas the physiological studies (Garside \& Doran, 2000; Reiser et al., 2001; Welbergen \& Clijsen, 1990) had revealed a tendency toward a beneficial effect of a more extended body configuration, such a conclusion cannot be drawn from the present study.

In six of the seven muscle in which muscle activity was affected, activity increased in the backward extended configuration. But as the muscle lengths in most of these muscles have been found to vary also with body configuration, an 
increase of muscle activity cannot be interpreted as increased effort. However, from the above analysis it can be concluded that manipulation of the trunk angle, through changes in muscle activity and in the association between muscle activation and changes in muscle length, affects muscle dynamics and force production.

Comparing the EMG changes reported in the present study to those presented for other manipulations which are known to affect performance considerably, it becomes clear that the effects of trunk angle manipulations are considerable. Effects on EMG activity found in this study exceed the EMG adaptations reported on manipulation of chain ring (Neptune \& Herzog, 2000) and on differences between sitting and standing or uphill riding (Li \& Caldwell, 1998). EMG amplitude changes found in the present study were of the same magnitude as those assessed for manipulation of cadence (Neptune et al., 1997) and of body orientation (Brown et al., 1996). So far trunk angle has merely been considered as an aerodynamic factor. But these comparisons indicate that trunk angle is a factor in producing propulsive power and is thus relevant in bicycle design and cycling performance.

The study shows that adaptations to changed lengths of muscles proximal in the multi-body system occur throughout the entire chain of segments. Changes in the activity of the directly affected muscles of the hip joint are not surprising. In the knee joint region the adaptations were found to be minimal; the knee joint kinematics appeared to be robust, and the activity of the monoarticular knee joint muscle considered was not affected. Around the ankle joint, adaptations were seen in kinematics and in muscle activity. Changing the lengths of muscles affects their ability to generate force and to contribute to a joint moment. But adjusting the degree of activity of these muscles (EMG) can partly compensate for this. If such compensation is not possible, torques at involved joints will be affected. In the case of a monoarticular muscle, the torque at one joint is directly affected.

Van Ingen Schenau et al. (1992) showed that in a multi-body system the control of external forces (e.g., pedal force) depends on the distribution of torques over adjacent joints. Consequently, affecting the torque in one joint will lead to adaptations around adjacent joints in order to maintain or to optimize the magnitude and direction of the external force. In the case of a biarticular muscle, an inadequate adaptation of muscle activity to changes in muscle length will directly affect torques at two joints. If a local adaptation had been possible in the present case, only activity changes in the monoarticular muscle spanning the hip joint would have been expected. The chain of adaptations found indicates that the limb is not able to react locally to applied manipulation. Although we cannot yet explain the specific adaptations found, it should be concluded that manipulation of one joint in a multi-body system can upset kinematics and muscle coordination throughout that entire system.

Theoretically, this conclusion can be deduced from the work of Van Ingen Schenau et al. (1992). However, to our knowledge, practical consequences have not yet been demonstrated. Compared to variations in body configurations that are seen in commonly available bicycles, both upright and recumbents, the trunk angle variations applied in this study are not excessive. From the present study it can be concluded that standard upright and crouched racing positions, and also various alternative recumbent positions, lying or sitting, will differ with respect to muscle coordination. The conclusion that local manipulations can have more global effects on movement can most likely be generalized to domains beyond cycling and 
sports. It will also be applicable to other fields of human movement in which muscle coordination is important and may affect performance (e.g., ergonomics, design of rehabilitation aids).

In this study we considered the effect of similar muscles operating at different lengths. But in human movement the opposite often occurs as well: muscles with different properties (i.e., different optimal lengths) being used under similar conditions. Different quadriceps heads of runners and cyclists are known to have different optimal lengths (Herzog, Guimaraes, Anton, \& Carter-Erdman, 1991; Savelberg \& Meijer, 2003). Combining this knowledge with the results of the present study, it can be hypothesized that differently trained subjects (e.g., runners vs. cyclist) perform a similar motor task (running or cycling) differently.

With respect to the effect of the manipulation, it is reasonable to assume that the magnitude of the local manipulation determines whether and where adaptation occurs in a multi-body system. Insight into the joint and muscle dynamics underlying the adaptations in muscle recruitment and joint kinematics will be needed in order to understand the relationship between local manipulation and global adaptation.

In conclusion, this study showed that changing trunk angle in cycling affects muscle recruitment, joint kinematics, and the association between muscle recruitment and muscle length. Evidence is presented that these adaptations will concur with adaptation in muscle and joint dynamics, and that as a consequence, trunk angle manipulation can be a factor in cycling performance and bicycle design. It is likely that the conclusions of this study can be generalized to other fields of human movement.

\section{References}

Brown, D.A., Kautz, S.A., \& Dairaghi, C.A. (1996). Muscle activity patterns altered during pedalling at different body orientations. Journal of Biomechanics, 29, 1349-1356.

Ericson, M.O., Nisell, R., Arborelius, U.P., \& Ekholm, J. (1985). Muscular activity during ergometer cycling. Scandinavian Journal of Rehabilitation Medicine, 17, 53-61.

Garside, I., \& Doran, D.A. (2000). Effect of bicycle frame ergonomics on triathlon km running performance. Journal of Sports Science, 18, 825-833.

Gregor, S.M., Perell, K.L., Rushatakankovit, S., Miyamoto, E., Muffoletto, R., \& Gregor, R.J. (2002). Lower extremity general muscle moment patterns in healthy individuals during recumbent cycling. Clinical Biomechanics, 17, 123-129.

Gross, A.C., Kyle, C.R., \& Malewicki, D.J. (1983). The aerodynamics of human-powered land vehicles. Scientific American, 249, 142-152.

Hawkins, D., \& Hull, M.L. (1991). A method for determining lower extremity muscletendon lengths during flexion/extension movements. Journal of Biomechanics, 23, 487-494.

Herzog, W., Guimaraes, A.C., Anton, M.G., \& Carter-Erdman, K.A. (1991). Moment-length relations of rectus femoris of speed skaters/cyclist and runners. Medicine and Science in Sports and Exercise, 23, 1289-1296.

Jorge, M., \& Hull, M.L. (1986). Analysis of EMG measurements during bicycle pedalling. Journal of Biomechanics, 19, 683-694.

Li, L., \& Caldwell, G.E. (1998). Muscle coordination in cycling: Effect of surface incline and posture. Journal of Applied Physiology, 85, 927-934. 
MacIntosh, B.R., Neptune, R.R., \& Horton, J.F. (2000). Cadence, power, and muscle activation in cycle ergometry. Medicine and Science in Sports Exercise, 32, 1281-1287.

Marsh, A.P., \& Martin, P.E. (1995). The relationship between cadence and lower extremity EMG in cyclists and noncyclists. Medicine and Science in Sports and Exercise, 27, 217-225.

Neptune, R.R., \& Herzog, W. (2000). Adaptation of muscle coordination to altered task mechanics during steady-state cycling. Journal of Biomechanics, 33, 165-172.

Neptune, R.R., Kautz, S.A., \& Hull, M.L. (1997). The effect of pedaling rate on coordination in cycling. Journal of Biomechanics, 30, 1051-1058.

Reiser, R.F., Peterson, M.L., \& Broker, J.P. (2001). Anaerobic cycling power output with variations in recumbent body configuration. Journal of Applied Biomechanics, 17, 204-216.

Savelberg, H.H.C.M., \& Meijer, K. (2003) Contribution of mono- and biarticular muscles to extending knee joint moments in runners and cyclists. Journal of Applied Physiology, 94, 2241-2248.

Van Ingen Schenau, G.J., Boots, P.J.M., de Groot, G., Snackers, R.J., \& van Woensel, W.L.M. (1992). The constrained control of force and position in multi-joint movements. Neuroscience, 46, 197-207.

Welbergen, E., \& Clijsen, L.P.V.M. (1990). The influence of body position on maximal performance in cycling. European Journal of Applied Physiology, 61, 138-142. 